\title{
Multiplicity of Massive Stars
}

\author{
Thomas Preibisch, Gerd Weigelt \\ Max-Planck-Institut für Radioastronomie, Auf dem Hügel 69, D-53121 \\ Bonn, Germany \\ Hans Zinnecker \\ Astrophysikalisches Institut Potsdam, An der Sternwarte 16, D-14482 \\ Potsdam, Germany
}

\begin{abstract}
We discuss the observed multiplicity of massive stars and implications on theories of massive star formation. After a short summary of the literature on massive star multiplicity, we focus on the $\mathrm{O}$ and B-type stars in the Orion Nebula Cluster, which constitute a homogenous sample of very young massive stars. 13 of these stars have recently been the targets of a bispectrum speckle interferometry survey for companions. Considering the visual and also the known spectroscopic companions of these stars, the total number of companions is at least 14 . Extrapolation with correction for the unresolved systems suggests that there are at least 1.5 and perhaps as much as 4 companions per primary star on average. This number is clearly higher than the mean number of $\sim 0.5$ companions per primary star found for the low-mass stars in the general field population and also in the Orion Nebula cluster. This suggests that a different mechanism is at work in the formation of high-mass multiple systems in the dense Orion Nebula cluster than for low-mass stars.
\end{abstract}

\section{Introduction}

It is a well known fact that many, perhaps most stars in our galaxy are members of multiple systems. Although this paper is supposed to deal with massive stars $\left(M_{*} \geq 8-10 M_{\odot}\right)$, we will start with a brief look at the multiplicity of solar type stars, first because this is very well known, and second because it is important to compare the multiplicity of the massive stars to that of the solar type stars. The main characteristics of the multiplicity of solar type field stars can be summarized as follows (c.f. Duquennoy \& Mayor 1991): The binary frequency (i.e. the probability that a given object is multiple; cf. Reipurth \& Zinnecker 1993) is about $45 \%$, and any primary star has about 0.5 companions on average. The ratio of single: double: triple: quadruple systems is $57: 38: 4: 1$. The distribution of orbital periods follows a log-normal relationship, and the mass ratio distribution decreases for $q:=M_{2} / M_{1} \rightarrow 1$ and seems to be consistent with random pairing from the field star initial mass function. 
There are several reasons why the multiplicity of massive stars is less well known than for the low-mass stars. One of them is that massive stars are far less numerous than low-mass stars, and thus they are typically located at larger distances. Another problem is caused by the very high luminosities of massive stars, causing extreme brightness contrasts between the massive primary star and any low-mass companion, strongly hampering the detection of visual companions. However, since a good knowledge of the multiplicity of massive stars can yield very important constraints on high-mass stellar formation theories (see below), a study of the multiplicity of massive stars is highly desirable.

\section{Formation of Massive Stars}

The formation mechanism of massive stars is still not well understood (cf. Stahler et al. 1999). In contrast to low- and intermediate-mass stars, high-mass stars cannot form through gravitational collapse in molecular cloud cores and subsequent accretion, because, as soon as the stellar core reaches a mass of $\sim 10 M_{\odot}$, the radiation pressure on the infalling dust halts the accretion and thus limits the mass (Yorke \& Krügel 1977; Yorke 1993).

An alternative model for the formation of massive stars is based on collisions and subsequent mergers of low- or intermediate-mass protostars in the dense centers of young clusters. At first sight, the typical conditions in star forming regions (stellar number densities of $n_{*} \lesssim 10^{5}$ stars $\mathrm{pc}^{-3}$ and velocity dispersions of $\sigma_{v} \approx 2 \mathrm{~km} / \mathrm{sec}$ ) suggest extremely long collision time scales of $t_{\text {coll }} \gtrsim 10^{10}$ years. This seems to indicate that such models are not very reasonable. However, in a very young cluster there are important peculiarities that can dramatically change the picture. One aspect is that very young stars are typically surrounded by circumstellar disks or envelopes, making their geometrical cross section much larger than the stellar cross section. Another aspect is that the cross section can be strongly enhanced in moderately slow encounters by the gravitational focusing effect. Furthermore, it is important to take into account that during gravitational interactions stars do not behave like solid bodies: close stellar encounters (pericenter $\lesssim 4$ stellar radii) can induce huge tidal waves on the stars, dissipating kinetic energy into tidal heat; this eventually can lead to the formation of bound systems or even stellar mergers (cf. Mardling 1995).

Another crucial factor is that the forming star cluster not only contains young stars, but also an appreciable amount of dense gas, from which the forming stars accrete mass. Bonnell et al. (1998) have performed theoretical investigations and showed that the transfer of mass from diffuse gas to stars decreases the total energy of the cluster and also the stellar velocity dispersion. This leads to a strong shrinking of the cluster, which strongly boosts the stellar number density. Altogether these effects can cause the collision time scale to greatly decrease to values as low as $t_{\text {coll }} \approx 10^{5}$ years, making the collision and subsequent merger of low- or intermediate-mass protostars an apparently viable way to form massive stars, especially in the center of dense clusters.

If this mechanism is indeed the way how the majority of massive stars form, one would expect multiple systems to be very common amongst the massive stars, because many of the collisions will not lead to stellar mergers but to the 
formation of multiple systems. Observational multiplicity studies in very young clusters thus can help to test this theory.

\section{Observational Results on OB Star Multiplicity}

\subsection{Summary of Literature Results}

Many previous studies have investigated the multiplicity of massive stars. In the following short compilation, which is by no means meant to be complete, we summarize a few of the most important results.

Abt (1979) studied the multiplicity of early B-type (B2-B5) stars. By applying a correction for observational incompleteness he estimated that the true binary frequency among these stars might be as high as $\approx 65 \%$. He also found that the distribution of orbital periods is similar to that of solar-type binaries, and concluded that the binary characteristics of normal stars do not depend sensitively upon primary mass.

In a review paper, Abt (1983) concluded that the spectroscopic binary frequency among B-type stars seems to be higher than among F- and G-type stars. In a further study, Abt et al. (1990) searched for spectroscopic binaries among 116 B2-B5 stars. This study indicated that the number of companions is rather high: assuming that the companion mass function is equal to the Salpeter IMF, the correction for observational incompleteness suggested that there are at least 0.8 (1.9) companions with $M_{2} \geq 2 M_{\odot}\left(M_{2} \geq 1 M_{\odot}\right)$ per primary star on average. This would be much higher than the mean number of 0.5 companions per solar type field star primary.

Morrell \& Levato (1991) spectroscopically studied 96 OB stars in the Orion OB1 association and found that the percentage of spectroscopic binaries with periods of $P<100$ days is $32 \%$, significantly more than among solar type field stars. Garmany et al. (1980) derived a spectroscopic binary frequency of $36 \%$ among a sample of $67 \mathrm{O}$ stars. They found that $85 \%$ of the systems have mass ratios of $q>0.4$, i.e. most of the companions to these high-mass stars are again high-mass stars.

McAlister et al. (1993) performed speckle interferometric observations of a huge sample of 2088 OB stars, representing $23 \%$ of the members of the Bright Star Catalogue. Their observed binary frequency of B stars was $14 \%$.

Mason et al. (1998) performed a speckle survey among a magnitude limited $(\mathrm{V}<8)$ sample of 227 O-type stars. They detected 15 binaries in the range of separations $0.035^{\prime \prime}<\rho<1.5^{\prime \prime}$. Taking into account the previously known visual and spectroscopic companions, their results demonstrated that at least $60 \%$ of the O-type stars have companions. The distribution of mass ratios they derived is flat or decreasing for $\mathrm{q} \rightarrow 1$, in a notable contrast to the result of Garmany et al. (1980).

\subsection{General Conclusions on OB Star Multiplicity}

The above mentioned studies agree on the result that the multiplicity of OB stars is rather high, probably at least as high as for the solar-type field stars. The derived mass ratio distributions, however, differ strongly from study to study. 
Many of the studies mentioned above share a serious disadvantage by being based on rather inhomogeneous samples of stars. Despite the advantage of allowing to investigate a large number of stars, any magnitude limited sample is a mixture of stars of different distances, different ages, and different origin. This will inevitably cause very serious and complicated selection effects.

Therefore, it was our goal to study a more homogeneous sample of massive stars. A very good way to do this is to study the population of massive members of a nearby star forming region. In such a sample, all the stars have a common distance, age, and origin. Another important factor is that a very young cluster is especially well suited for the detection of binary companions since any lowor intermediate mass companion will still be in its pre-main sequence phase and thus typically a factor of $\sim 2-10$ brighter than on the main sequence. This significantly decreases the enormous difference in brightness between the luminous primary star and its low-mass companion, which usually makes the observational detection of the companion very difficult or even impossible.

\section{Multiplicity of the Massive Stars in the Orion Nebula Cluster}

The Orion Nebula Cluster (ONC) is a very promising target for a study of the multiplicity of massive stars. It is nearby $(450 \mathrm{pc})$, very young $(<1 \mathrm{Myr})$, and its high central stellar density $\left(n_{*} \sim 5 \times 10^{4} \mathrm{pc}^{-3}\right)$ suggests that stellar collisions might actually be important. Also, it is very well investigated (e.g. Genzel \& Stutzki 1989; Herbig \& Terndrup 1986; McCaughrean \& Stauffer 1994; Hillenbrand \& Hartmann 1998) and the stellar population is well known (cf. Brown et al. 1994; Hillenbrand 1997). The compilation of Hillenbrand (1997) lists 27 Oand B-type stars as ONC members; these stars constitute a homogenous sample of very young massive stars.

Several multiplicity studies have been performed for the low-mass stars in the ONC (cf. Padgett et al. 1997; Petr et al. 1998; Simon et al. 1999). These studies seem to agree that the multiplicity of the low-mass stars in the ONC is consistent with that in the general field, i.e. there is no evidence for an overabundance of multiple systems as has been found for the low-mass pre-main sequence stars in the Taurus region (c.f. Leinert et al. 1993, 1997; Köhler \& Leinert 1998; Ghez et al. 1997).

As far as the high-mass stars in the ONC are concerned, a number of searches for spectroscopic binaries have been performed (e.g. Abt et al. 1991; Morrell \& Levato 1991). The Trapezium stars have been observed with nearinfrared speckle holographic methods by Petr et al. (1998) and with adaptive optics methods by Simon et al. (1999). In order to get more complete information about the multiplicity of these stars, we have recently performed a bispectrum speckle interferometry (cf. Weigelt 1977) survey of 13 of the ONC O- and B-type stars at the SAO $6 \mathrm{~m}$ telescope (Weigelt et al. 1999; Preibisch et al. 1999). In combination with the information on the spectroscopic companions, this gives a comprehensive (though, of course, still not $100 \%$ complete) picture of the multiplicity of these stars. 


\subsection{Known Companions to the 13 Orion OB Stars}

In our speckle images 8 visual companions have been found in total. Considering both, the visual and the spectroscopic companions of the 13 target stars, the total number of companions is at least 14 . The properties of the known companions are summarized in Table 1.

Table 1. Summary of all known companions of the observed ONC stars. References: 1: Preibisch et al. (1999); 2: Weigelt et al. (1999); 3: Petr et al. (1998); 4: Bossi et al. (1989); 5: Simon et al. (1999); 6: Abt et al. (1991); 7: Levato \& Abt (1976).

\begin{tabular}{|lrr|lrlr|}
\hline $\begin{array}{c}\text { Primary } \\
\text { Par- }\end{array}$ & $\begin{array}{r}M_{1} \\
{\left[M_{\odot}\right]}\end{array}$ & Companion & $\begin{array}{c}\rho \\
{[\mathrm{AU}]}\end{array}$ & $q$ & Ref \\
\hline $1605-1$ & V372 Ori & 3.5 & -2 (spec) & & $\sim 0.95$ & 7 \\
\hline 1744 & HD 36981 & 4.8 & - & & & \\
\hline 1772 & LP Ori & 7.2 & - & & & \\
\hline $1863-1$ & $\theta^{1}$ Ori B & 7 & -2 (vis) & 430 & $\sim 0.22$ & 1,2 \\
& & & -3 (vis) & 460 & $\sim 0.10$ & 1,2 \\
& & & -4 (vis) & 260 & $\sim 0.03$ & 1,5 \\
& & & -5 (spec) & 0.13 & & 6 \\
\hline $1865-1$ & $\theta^{1}$ Ori A & 16 & -2 (vis) & 100 & $\sim 0.25$ & 2,3 \\
& & & -3 (spec) & 1 & $\sim 0.13$ & 4 \\
\hline 1889 & $\theta^{1}$ Ori D & 17 & - & & & \\
\hline $1891-1$ & $\theta^{1}$ Ori C & 45 & -2 (vis) & 16 & $\sim 0.12$ & 2 \\
\hline $1993-1$ & $\theta^{2}$ Ori A & 25 & -2 (vis) & 173 & $\sim 0.28$ & 1 \\
& & & -3 (spec) & 0.47 & $\sim 0.35$ & 6 \\
\hline 2031 & $\theta^{2}$ Ori B & 12 & - & & & \\
\hline $2074-1$ & NU Ori & 14 & -2 (vis) & 214 & $\sim 0.07$ & 1 \\
& & & -3 (spec) & 0.35 & $\sim 0.2$ & 6 \\
\hline $2271-1$ & HD 37115 & 5 & -2 (vis) & 400 & $\sim 0.29$ & 1 \\
\hline 2366 & HD 37150 & 15 & - & & & \\
\hline $2425-1$ & WH 349 & 4 & -2 (vis) & 388 & $\sim 0.04$ & 1 \\
\hline
\end{tabular}

With 14 known companions to the 13 target stars, the mean number of observed companions per primary star is 1.1. Although this clearly is a firm lower limit to the true number of companions, it is already $2 \times$ more than the average number of companions among the low-mass field star primaries.

\subsection{Estimation of the True Number of Companions}

Obviously, the number of known companions is only a lower limit to the true number of companions, as any observational search for companions is subject to limited sensitivity. Preibisch et al. (1999) estimated the fraction of companions 
that might have been not detected in the speckle observations due to their being either too close to be resolved ( $\rho \lesssim 35$ mas) or too faint to be detected $(\Delta K \gtrsim 4$ $\mathrm{mag}$ ). The correction factor, i.e. the ratio of all companions to detected companions, was estimated to be between 2.5 and 6.7, depending on the assumptions about the underlying mass ratio distribution and the distribution of separations. Since the speckle observations revealed 8 visual companions to 13 target stars, the extrapolated number of companions is probably in the range $\approx[20 \ldots 50]$. This suggests that there are $\approx[1.5 \ldots 4]$ companions per primary star on average, and this is at least $3 \times$ more than for low-mass primaries.

\subsection{Properties of the Multiple Systems}

\section{- Distribution of mass ratios}

While the mass ratio distribution for low-mass binaries is rather well known to be consistent with the field IMF, several very different distributions have been derived for the mass ratios in different samples of high-mass binary systems. The results range from distributions favoring low-mass companions (e.g. Mason et al. 1998), over flat distributions, to distributions which more or less strongly favor relatively massive companions (e.g. Abt \& Levy 1978; Garmany et al. 1980). In our sample, all known companions are low- or intermediate mass stars and the distribution of mass ratios has a strong peak at moderately low values.

In Fig. 1 we compare our empirical mass ratio distribution with the following different models:

(a) a mass ratio distribution given by random pairing of stars drawn from the Scalo (1998) field star IMF;

(b) a flat distribution of mass ratios, i.e. $f(q)=$ const. for $[0 \leq q \leq 1]$;

(c) a distribution that slightly favors massive companions, $f(q) \propto q^{0.25}$ for $[0 \leq q \leq 1]$, as derived by Abt \& Levy (1978) for a sample of early B-type stars;

(d) a distribution of mass ratios which strongly favors systems with (nearly) equal masses. Here we consider the findings of the binary survey among O-type stars performed by Garmany et al. (1980), which can be roughly approximated by a half Gaussian distribution with a peak at $q=1$ and a width of $\sigma \approx 0.45$ for $[0 \leq q \leq 1]$;

It is rather obvious that our empirical distribution is not consistent with the models (c) and (d). Thus we conclude that any distribution favoring high mass ratios seems to be definitely excluded by our data. Furthermore, one has to keep in mind that most probably there are more, still undetected faint low- $q$ companions, which are missing in our empirical distribution. Even if we take into account that our estimates of the mass ratios are subject to significant uncertainties (see Preibisch et al. 1999 for a detailed discussion), it appears very likely that the true mass ratio distribution is significantly peaked towards moderately low mass ratios.

\section{- Number of components per system}

Among the solar type field stars only $\mathbf{5 \%}$ of the systems are triple or higherorder systems (Duquennoy \& Mayor 1991). In our sample, however, 31\% of the systems have at least 3 components. The numbers of systems with given order are compared to the corresponding numbers for solar type field stars in Table 


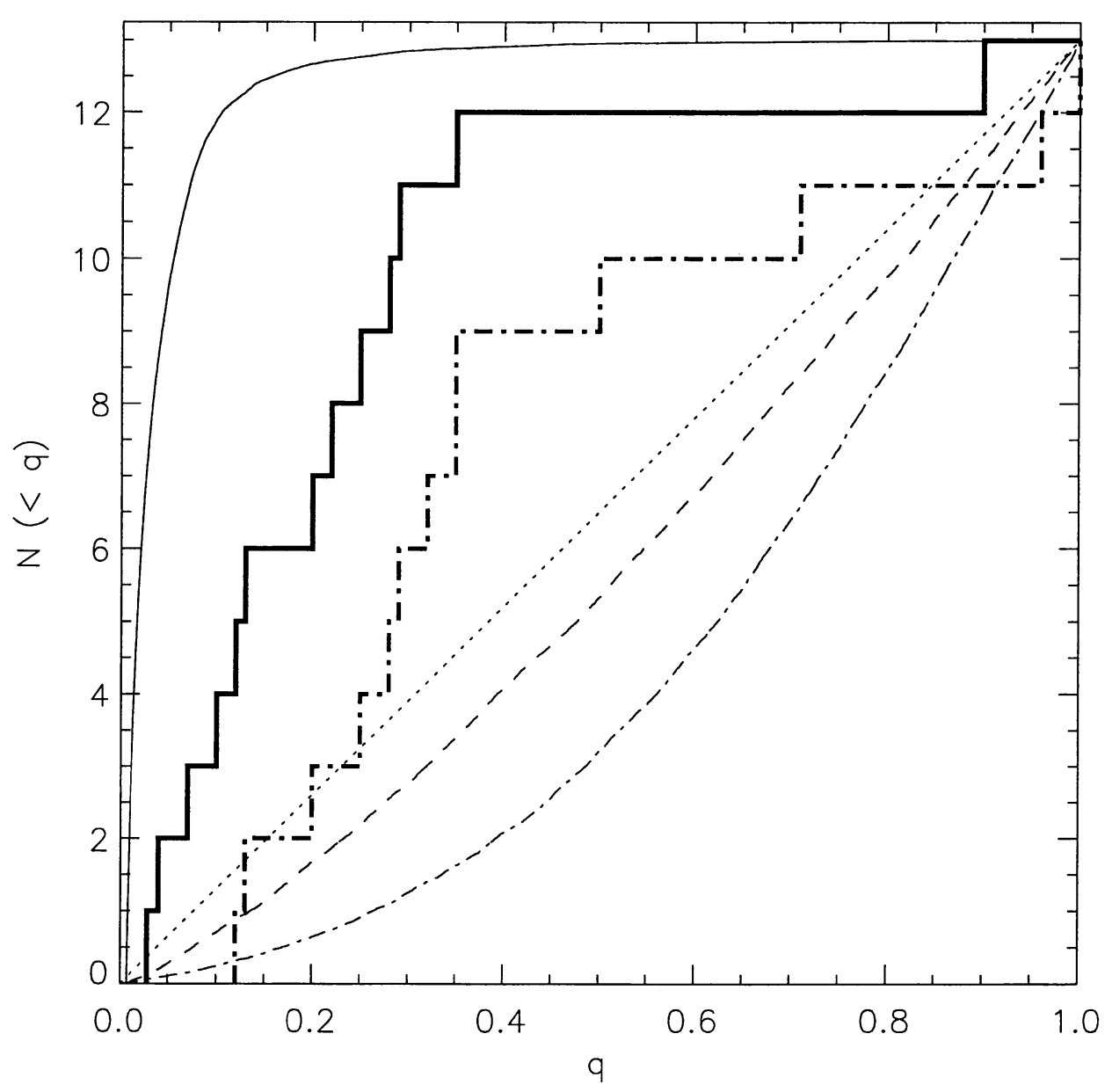

Figure 1. The thick solid line shows the cumulative distribution function of observed mass ratios of the ONC multiple systems, based on our best estimates of the companion masses. The thick dashed-dotted line shows the same distribution based on very conservative upper mass limits for the companions (for details see Preibisch et al. 1999). These empirical distributions are compared to the four different model distributions described in the text: (a): random pairing from the Scalo (1998) field star IMF (thin solid line); (b): flat mass ratio distribution (thin dotted line); (c): a distribution that slightly favors massive companions (dashed line); (d): a distribution that strongly favors systems with (nearly) equal masses (thin dashed-dotted line). 


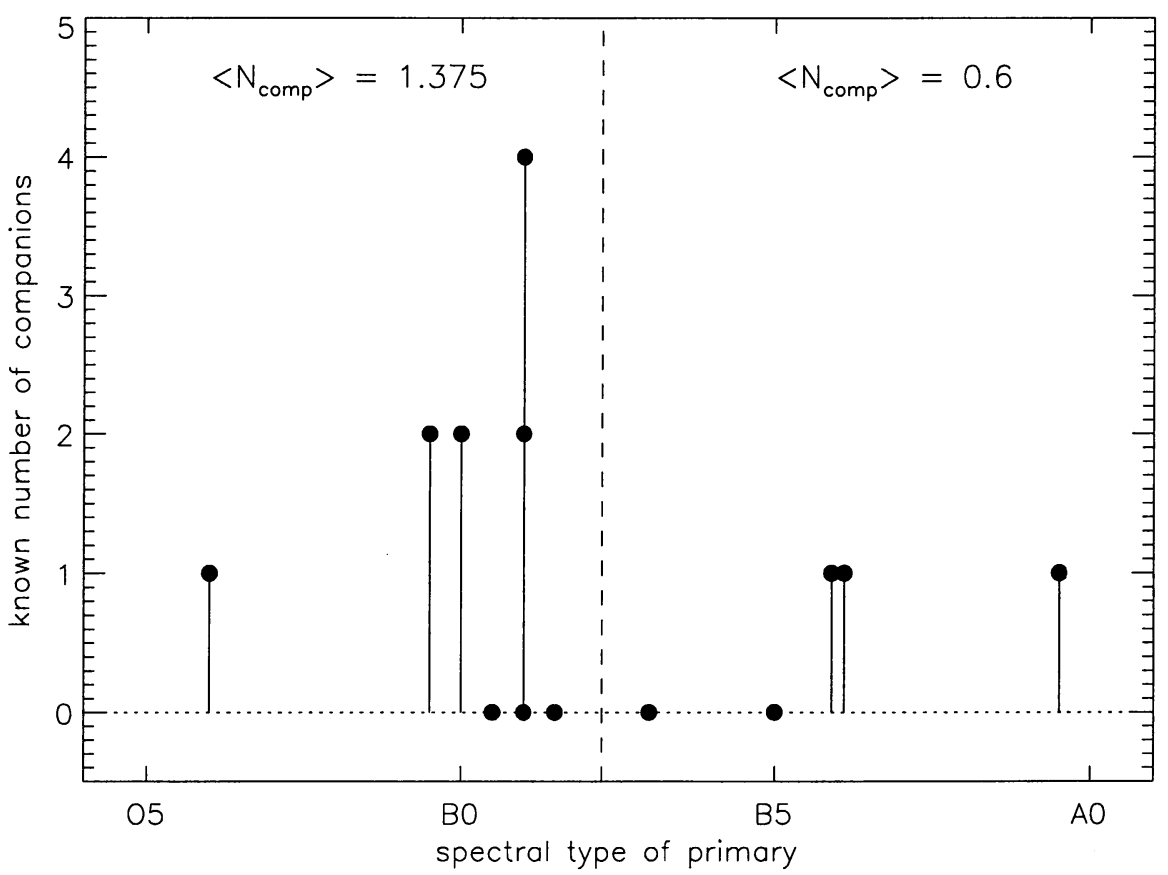

Figure 2. The known number of companions is plotted against the spectral type of the primary.

2. One can clearly see that the fraction of higher-order multiple systems seems to be enhanced among the OB stars.

Table 2. Systems with multiplicity of a given order in our sample and among the solar type field stars. Data for the solar type field stars are from Duquennoy \& Mayor (1991).

\begin{tabular}{|l|cccc|}
\hline & singles & binaries & triples & quadruples \\
\hline number & 5 & 4 & 3 & 1 \\
fraction & $38 \pm 13 \%$ & $31 \pm 13 \%$ & $23 \pm 12 \%$ & $8 \pm 7 \%$ \\
\hline solar type stars & $57 \pm 4 \%$ & $38 \pm 4 \%$ & $4 \pm 1 \%$ & $1 \pm 1 \%$ \\
\hline
\end{tabular}

\section{- Multiplicity and primary spectral type}

A remarkable trend for a higher degree of multiplicity among the stars of very early spectral type as compared to the later type stars is apparent in our sample. The average number of known (visual \& spectroscopic) companions per primary is 2.3 times higher among the primaries with spectral type earlier than B3 (11 known companions to 8 primaries) than among the later type primaries ( 3 known companions to 5 primaries). 


\section{Conclusions}

Our results show that the multiplicity of the massive stars in the Orion Nebula Cluster is very high. Even if we only consider the already known companions, there are on average at least 1.1 companions per high-mass primary star, about twice as many as found for low-mass primary stars in the general field as well as in the Orion Nebula Cluster. If we correct these numbers for observational incompleteness, i.e. for the still undetected companions, our data suggest that there should be [1.5 ..4] companions per primary star on average, i.e. $\sim[3 \ldots 8] \times$ more than for low-mass primaries. Another important difference between the massive stars in the Orion Nebula Cluster and the low-mass stars is the higher fraction of higher-order multiple systems (triples, quadruplets, ...) among the OB stars. These findings strongly suggest a different formation mechanism for high-mass and low-mass multiple systems.

We also find a trend that $\mathrm{O}$ - and early B-type stars have more companions than later B-type stars. The nature of our results seems to support the idea that high-mass stars form through collisions of protostars. However, at least in its present state, this theory provides no detailed predictions of the properties of the multiple systems. If the merging objects would be just spherical stars, one would expect most of the multiple systems to have quite small separations, probably well below the resolution limit of the speckle observations ( $\sim 20 \mathrm{AU})$. However, the merging protostars are surrounded by extended disks and/or envelopes, and it is not yet clear how this changes the outcome of the collision and merging processes.

Furthermore, the dynamical evolution of a cluster will also affect the properties of the multiple systems. For example, wide binaries are readily destroyed by stellar encounters in the dense cluster (e.g. Bonnell 2000). Recent model calculation by Kroupa et al. (1999) indicate that, under certain circumstances, cluster dynamical evolution can noticeably change the properties of the multiple systems on time scales as short as $\lesssim 1$ Myr. This suggests that even in the very young Orion Nebula Cluster the observed properties of the multiple systems might not necessarily be identical to the primordial multiplicity.

Clearly, further work is necessary for the theoretical as well as the observational side of the topic. As far as observations are concerned, we note that we have already performed speckle interferometric observations of the other $14 \mathrm{OB}$ stars in the Orion nebula cluster. The data analysis will soon be completed and we should then be able to draw more firm conclusions on the basis of the full sample of all 27 OB stars in the Orion Nebula Cluster.

\section{References}

Abt, H. A. 1983, ARAA, 21, 343

Abt, H. A., Gomez, A. E., \& Levy, S. G. 1990, ApJS, 74, 551

Abt, H. A., Wang, R., \& Cardona, O. 1991, ApJ, 367, 155

Bonnell, I. A. 2000, in ASP Conf. Ser. Vol. 198, Stellar Clusters and Associations: Convection, Rotation, and Dynamos, ed. R. Pallavicini, G. Micela \& S. Sciortino, (San Francisco: ASP), 161

Bonnell, I. A., Bate, M. R., \& Zinnecker, H. 1998, MNRAS, 298, 93 
Bossi, M., Gaspani, A., Scardia, M., \& Tadini, M. 1989, A\&A, 222, 117

Brown, A. G. A., de Geus, E. J., \& de Zeeuw, P. T. 1994, A\&A, 289, 101

Duquennoy, A., \& Mayor, M. 1991, A\&A, 248, 485

Fischer, D. A., \& Marcy, G. W. 1992, ApJ, 396, 178

Genzel, R., \& Stutzki, J. 1989, ARA\&A, 27, 41

Ghez, A. M., McCarthy, D. W., Patience, J. L., Beck, T.L. 1997, ApJ, 481, 378

Herbig, G. H., \& Terndrup, D. M. 1986, ApJ, 307, 609

Hillenbrand, L. A. 1997, AJ, 113, 1733

Hillenbrand, L. A., \& Hartmann, L. W. 1998, ApJ, 492, 540

Hillenbrand, L. A., Strom, S. E., et al. 1998, AJ, 116, 1816

Kenyon, S. J., \& Hartmann, L. W. 1995, ApJS, 101, 117

Köhler, R., \& Leinert, Ch. 1998, A\&A, 331, 977

Kroupa, P., Petr, M. G., \& Mc Caughrean, M. J. 1999, NewAstr, 4, 495

Leinert, Ch., Zinnecker, H., Weitzel, N., et al. 1993, A\&A, 278, 129

Leinert, Ch., Richichi, A., \& Haas, M. 1997, A\&A, 318, 472

Levato, H., \& Abt, H. A. 1976, PASP, 88, 712

Mardling, R. A. 1995, ApJ, 450, 732

Mason, B. D., Gies, D. R., Hartkopf, W. I., et al. 1998, AJ, 115, 821

McCaughrean, M. J., \& Stauffer, J. R. 1994, AJ, 108, 1382

Morrell, N., \& Levato, H. 1991, ApJS, 75, 965

Padgett, D. L., Strom, S. E., \& Ghez, A. 1997, AJ, 477, 705

Petr, M. G., Du Foresto, V., \& Beckwith, S. V. W. 1998, ApJ, 500, 825

Preibisch, Th., Balega, Y., Hofmann, K.-H., Weigelt, G., \& Zinnecker, H. 1999, NewAstr, 4, 531

Prosser, C. F., Stauffer, J. R., Hartmann, L., et al. 1994, ApJ, 421, 517

Reipurth, B., \& Zinnecker, H. 1993, A\&A, 278, 81

Scalo, J. 1998, in ASP Conf. Ser. Vol. 142, The Stellar Initial Mass Function, ed. G. Gilmore \& D. Howell, (San Francisco: ASP), 201

Schultz, A. S. B., Colgan, S. W. J., Erickson, E. F., et al. 1999, ApJ, 511, 282

Simon, M., Close, L. M., \& Beck, T. L. 1999, AJ, 117, 1375

Stahler, S. W., Palla, F., \& Ho, P. T. P. 1999, in Protostars and Planets IV, ed. V. Mannings, A. Boss \& S. Russel, (Univ. of Arizona Press), in press

Walter, F. M., Alcalá, J. M., Neuhäuser, R., Sterzik, M., \& Wolk S. J. 1999, in Protostars and Planets IV, ed. V. Mannings, A. Boss \& S. Russel, (Univ. of Arizona Press), in press

Weigelt, G. 1977, Opt Commun., 21, 55

Weigelt, G., Balega, Y., Preibisch, Th., Schertl, D., Schöller, M., \& Zinnecker, H. 1999, A\&A, 347, L15

Yorke, H. W. 1993, in ASP Conf. Ser. Vol. 35, Massive Stars, Their Lives in the Interstellar Medium, ed. J. Cassinelli \& E. Churchwell, (San Francisco: ASP), 45

Yorke, H. W., \& Krügel, E. 1977, A\&A, 54, 183 